\title{
Synthesis and electrical properties of $\mathrm{BaBiO}_{3}$ and high resistivity $\mathrm{BaTiO}_{3}-\mathrm{BaBiO}_{3}$ ceramics
}

\author{
Nitish Kumar* ${ }^{\dagger}, \S$, Stephen L. Golledge ${ }^{\star}$ and David P. Cann* \\ *Materials Science, School of Mechanical Industrial, and \\ Manufacturing Engineering \\ Oregon State University, Corvallis, Oregon 97331, USA \\ ${ }^{\dagger}$ School of Materials Science and Engineering \\ The University of New South Wales \\ New South Wales 2052, Australia \\ ${ }^{\star}$ CAMCOR Surface Analytical Facility \\ University of Oregon, Eugene \\ Oregon 97403, USA \\ \$nitishkumar.iitk@gmail.com
}

\begin{abstract}
Received 25 September 2016; Revised 13 November 2016; Accepted 14 November 2016; Published 16 December 2016
Ceramics of the composition $\mathrm{BaBiO}_{3}(\mathrm{BB})$ were sintered in oxygen to obtain a single phase with monoclinic $I 2 / m$ symmetry as suggested by high-resolution X-ray diffraction. X-ray photoelectron spectroscopy confirmed the presence of bismuth in two valence states $-3+$ and 5+. Optical spectroscopy showed presence of a direct bandgap at $\sim 2.2 \mathrm{eV}$ and a possible indirect bandgap at $\sim 0.9 \mathrm{eV}$. This combined with determination of the activation energy for conduction of $0.25 \mathrm{eV}$, as obtained from $a c$ impedance spectroscopy, suggested that a polaron-mediated conduction mechanism was prevalent in BB. The BB ceramics were crushed, mixed with $\mathrm{BaTiO}_{3}(\mathrm{BT})$, and sintered to obtain $\mathrm{BT}-\mathrm{BB}$ solid solutions. All the ceramics had tetragonal symmetry and exhibited a normal ferroelectric-like dielectric response. Using $a c$ impedance and optical spectroscopy, it was shown that resistivity values of BT-BB were orders of magnitude higher than BT or BB alone, indicating a change in the fundamental defect equilibrium conditions. A shift in the site occupancy of Bi to the A-site is proposed to be the mechanism for the increased electrical resistivity.

Keywords: $\mathrm{BaBiO}_{3}$; $\mathrm{BaTiO}_{3}$; conduction mechanism; transport properties; synchrotron; X-ray photoelectron spectroscopy; impedance spectroscopy; defects.
\end{abstract}

\section{Introduction}

The $\mathrm{BaBiO}_{3}(\mathrm{BB})$ has a distorted perovskite crystal structure with an ordered alternating $\mathrm{Bi}^{3+}$ and $\mathrm{Bi}^{5+} .1,2$ At room temperature, this charge disproportionation and ordering coupled with tilting of $\mathrm{BiO}_{6}$ corner-shared octahedra, result in a cell with monoclinic symmetry $(I 2 / m) \cdot{ }^{1,3}$ It was reported that BB only has out-of-phase tilting, denoted by $a^{0} b^{-} b^{-}$and $a^{-} a^{-} a^{-}$in Glazer's notation. ${ }^{4,5}$ There have been a number of reports on the compound $\mathrm{BB}$, especially polycrystalline $\mathrm{BB}$ ceramics doped with acceptors such as potassium, lead, and other additives, for potential use in high-temperature superconductor applications. ${ }^{6-13}$ The transition from semiconducting behavior to superconducting behavior occurs with doping and the critical temperature $\left(T_{c}\right)$ typically depends upon the doping concentration and type. ${ }^{6,7}$ It has been suggested that a phonon-mediated electron pairing mechanism for superconductivity may be significant, rather than Bardeen-Cooper-Schrieffer (BCS) theory. ${ }^{14}$

While doped BB has been studied primarily for superconductor applications as mentioned above, $\mathrm{BaBiO}_{3}$-doped $\mathrm{BaTiO}_{3}$ (BT-BB) solid solutions have been studied by some researchers for use as positive temperature coefficient of resistivity (PTCR) devices. ${ }^{15,16}$ In one study, it was shown that addition of BB increased the Curie point so that the PTCR effect was initiated above $\sim 150^{\circ} \mathrm{C} .{ }^{17}$

This report is focused on BT-BB solid solutions for the purpose of understanding the influence of $\mathrm{BB}$ additions on the electrical properties of BT. It has been demonstrated that small concentration of $\mathrm{BB}$ were enough to increase the resistivity of BT-BB solid solutions by several orders of magnitude, suggesting a drastic change in underlying defect chemistry. This is an important result because high resistivity will enable devices made using these ceramics to be used under high temperature and high electric fields. Application of higher electric fields is also important to increase energy density values of ceramic capacitors. ${ }^{18}$ At the same time, it may also help understand the defect chemistry of $\mathrm{BT}-\mathrm{Bi}(\mathrm{Me})$ $\mathrm{O}_{3}$ class of ceramics (excellent candidates for high temperature and high energy density dielectrics. ${ }^{19,20}$ ) as BB has been found to exist as an intermediate phase during processing and can potentially influence defect equilibrium conditions owing to multivalent bismuth. ${ }^{21}$ In this paper, BT-BB

This is an Open Access article published by World Scientific Publishing Company. It is distributed under the terms of the Creative Commons Attribution 4.0 (CC-BY) License. Further distribution of this work is permitted, provided the original work is properly cited. 
solid solutions were prepared with small mole fractions of BB to study the resultant dielectric and transport properties. This work also includes a full structural characterization of the BB and BB-BT ceramics prepared in this study.

\section{Experimental Procedure}

Solid-state methods was used to prepare $\mathrm{BB}$ ceramics using the precursors $\mathrm{BaCO}_{3}$ (Alfa Aesar, >99.8\%) and $\mathrm{Bi}_{2} \mathrm{O}_{3}$ (Aldrich, $>99.9 \%$ ). The powders were mixed together in the proper stoichiometric ratio, ground using a vibratory mill for $6 \mathrm{~h}$ and dried overnight in an oven $\left(\sim 80^{\circ} \mathrm{C}\right)$. The powders were then calcined in alumina crucibles at $725^{\circ} \mathrm{C}$ for $24 \mathrm{~h}$ and then milled with $\sim 3 \mathrm{wt} . \%$ Rhoplex binder and dried again. Green pellets in the shape of thin discs were prepared by cold-pressing uniaxially at a pressure of $\sim 200 \mathrm{MPa}$ and then sintered in oxygen at $825^{\circ} \mathrm{C}$ for $12 \mathrm{~h}$. It was difficult to achieve single phase on sintering in air, as will be seen later. To make solid solutions of $\mathrm{BT}$ and $\mathrm{BB}$, the sintered $\mathrm{BB}$ pellets were ground into powder form and then mixed with appropriate amounts of BT (Sakai Chemical Industry). The mixture was then milled with $\sim 3$ wt.\% Rhoplex binder and dried. The green pellets were prepared using similar conditions as described above, and then sintered in air on a bed of calcined powder in closed alumina crucibles. The compositions investigated were $(1-x) \mathrm{BT}-x \mathrm{BB}$, where $x=0.001$, $0.003,0.005,0.01$, and 0.02 , and will be henceforth abbreviated as $0.1 \mathrm{BB}, 0.3 \mathrm{BB}, 0.5 \mathrm{BB}, 1 \mathrm{BB}$, and $2 \mathrm{BB}$, respectively. These compositions were sintered at $1050^{\circ} \mathrm{C}$ for $4 \mathrm{~h}, 1000^{\circ} \mathrm{C}$ for $4 \mathrm{~h}, 950^{\circ} \mathrm{C}$ for $4 \mathrm{~h}, 990^{\circ} \mathrm{C}$ for $8 \mathrm{~h}$, and $990^{\circ} \mathrm{C}$ for $8 \mathrm{~h}$, respectively. For purposes of comparison, some bismuthdoped ceramics were also prepared with $0.3 \mathrm{~mol} \% \mathrm{Bi}$ (or $0.15 \mathrm{~mol} \% \mathrm{Bi}_{2} \mathrm{O}_{3}$ ) and $0.5 \mathrm{~mol} \% \mathrm{Bi}$ (or $0.25 \mathrm{~mol} \% \mathrm{Bi}_{2} \mathrm{O}_{3}$ ) added to BT. For this, excess $\mathrm{Bi}_{2} \mathrm{O}_{3}$ was added to BT powders, so as to achieve the above-mentioned doping levels. The powders were milled with $3 \mathrm{wt} . \%$ binder, dried, and then green pellets were sintered at $1000^{\circ} \mathrm{C}$ for $4 \mathrm{~h}$ and $950^{\circ} \mathrm{C}$ for $4 \mathrm{~h}$, respectively, on a bed of calcined powders. All the sintered pellets were polished to sub-millimeter thicknesses.

X-ray diffraction (XRD) (Bruker AXS D8 Discover, Madison, WI, USA) was performed on ground pellets for phase and crystal structure determination. High-resolution synchrotron powder diffraction data were collected on BB powders using beamline 11-BM at the Advanced Photon Source (APS), Argonne National Laboratory. An appropriate amount of the sample was loaded into a Kapton tube, sealed and mounted on the base provided by the 11-BM beamline of the APS. The diffraction experiments were carried out at room temperature using monochromatic synchrotron radiation of wavelength $0.413639 \AA$ and a step size of $0.001^{\circ}$. The crystal structure was refined using the Rietveld refinement program GSAS-II. ${ }^{22}$ The Chebyschev profile was used to fit the background and the number of background parameters used was 10. Parameters refined included peak-shaped parameters, scale, lattice parameters, atomic positions, isotropic displacement parameters, thermal parameters, and others.

Prior to any electrical measurements, $\mathrm{Ag}$ electrodes were applied on both sides of the pellets and then fired at $700^{\circ} \mathrm{C}$ for $30 \mathrm{~min}$. For undoped $\mathrm{BB}$ ceramics, instead of $\mathrm{Ag}, \mathrm{Pt}$ electrodes were used and with a firing temperature of $700^{\circ} \mathrm{C}$ for $2 \mathrm{~h}$ in oxygen. The pellets were placed in a high temperature measurement cell (NorECs AS ProbostatTM, Oslo, Norway) or low temperature environmental chamber (Delta Design 9023, California, USA) and the dielectric properties were measured using an LCR meter (Agilent 4284A, Santa Clara, CA, USA). An impedance analyzer (Solartron SI1260A equipped with Solartron 1296A dielectric interface, Farnborough, UK) was used to measure the impedance in the frequency range $0.1 \mathrm{~Hz}$ to $1 \mathrm{MHz}$. The SMaRT impedance measurement software program was used to collect the data. The optical measurements were carried out on crushed sintered pellets over the wavelength range of $200-1100 \mathrm{~nm}$ at room temperature using an Ocean Optics HR4000 UV-Vis Spectrometer (Ocean Optics, Dunedin, FL) with a balanced deuterium/tungsten halogen source. X-ray Photoelectron Spectroscopy (XPS) spectra from the BB ceramics were acquired on a ThermoScientific ESCALAB 250 spectrometer located at the Center for Advanced Materials Characterization in Oregon (CAMCOR). Photoemission was stimulated by a beam of monochromatized $\mathrm{Al} \mathrm{K} \alpha \mathrm{X}$-rays with a nominal spot size at the sample surface of $500 \mu \mathrm{m}$. A low energy electron source was used for charge neutralization. The spectra were acquired at a pass energy of $20 \mathrm{eV}$ (high-resolution spectra) or $150 \mathrm{eV}$ (survey spectra). Data analysis, including peak fitting, was performed with Avantage software, provided by the manufacturer.

\section{Results and Discussion}

\subsection{BB ceramics}

Figure 1(a) shows the comparison between XRD patterns for the samples sintered in oxygen and air for the same temperature and time. The pellet which was sintered in air had much higher concentration of secondary phases and underscored the importance of oxygen partial pressure during processing of BB. The pellets were suitably dense after sintering and had density values greater than $94 \%$ of theoretical value, as measured via Archimedes' method. Figure 1(b) shows the room temperature high-resolution XRD pattern of BB sintered in oxygen, which was performed to confirm the phase purity and formation of BB. Even within the detection limits of synchrotron radiation, no evidence of secondary phases was observed. Consistent with literature reports, a distorted perovskite structure with a monoclinic unit cell $(I 2 / \mathrm{m})$ was used to fit the pattern. ${ }^{3,23,24}$ The refinement resulted in a reasonably good fit can be seen in the difference curve in Fig. 1(b) (wR $=8.7 \%$ ). The lattice parameters obtained were $a=6.1865$ (7) $\AA, b=6.1399(4) \AA, c=8.6705(0) \AA$, and $\beta=90.178^{\circ}$, 


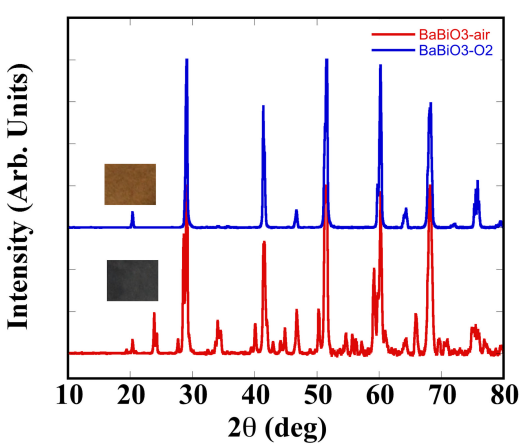

(a)

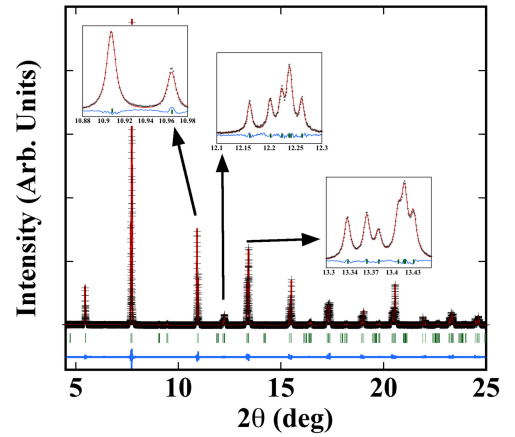

(b)

Fig. 1. (Color online) (a) XRD pattern of BB sintered in air (bottom) and oxygen (top). The square boxes show the color of the pellets after being sintered in air and oxygen. (b) Rietveld refinement of a high-resolution XRD pattern of BB sintered in oxygen. The data presented with $(+)$ symbol is the observed data and the black curves are calculated intensities. The bottom-most curve (blue) is the difference between observed and calculated intensities.

and Table 1 shows the parameters obtained from the refinement - atomic coordinates, site occupancy, and isotropic thermal parameters. All these parameters were within reasonable limits and corroborated the choice of unit cell and confirmed the phase purity of $\mathrm{BB}$ to be used later for fabricating the BT-BB solid solutions.

Even though bismuth with two different valence states $(+3$ and +5 in Table 1$)$ were used for the structural refinement above, XRD may not be sensitive enough to properly differentiate between trivalent $\mathrm{Bi}^{3+}$ and pentavalent $\mathrm{Bi}^{5+}$, which are the expected valence states of bismuth in $\mathrm{BB} .{ }^{23}$ To investigate the valence state of bismuth in BB, XPS was performed and the results have been shown in Fig. 2, where

Table 1. Parameters for $I 2 / m$ space group from the Rietveld refinement.

\begin{tabular}{lccccc}
\hline & $x$ & $y$ & $z$ & Occupancy & Uiso $\left(\AA^{2}\right)$ \\
\hline $\mathrm{Ba}$ & $0.4969(34)$ & 0 & $0.2512(84)$ & $0.999(3)$ & $0.0039(6)$ \\
$\mathrm{Bi}(1)$ & 0 & 0 & 0 & $1.021(6)$ & $0.0001(1)$ \\
$\mathrm{Bi}(2)$ & 0 & 0 & 0.5 & $1.023(4)$ & $0.0001(8)$ \\
$\mathrm{O}(1)$ & $-0.637(33)$ & 0 & $0.2420(46)$ & 1 & $0.0119(5)$ \\
$\mathrm{O}(2)$ & $0.2372(31)$ & $0.2381(66)$ & $0.0316(85)$ & 1 & $0.0131(0)$ \\
\hline
\end{tabular}

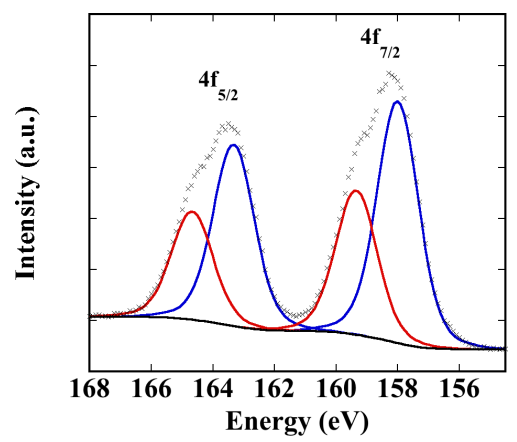

Fig. 2. (Color online) XPS on powdered BB ceramics. The data shown with " $\mathrm{x}$ " symbols are actual data obtained from the experiment. The background and fitted peaks have also been indicated. The low intensity fitted peaks (red) are from $\mathrm{Bi}^{5+}$. the $\operatorname{Bi}\left(4 \mathrm{f}_{5 / 2}, 4 \mathrm{f}_{7 / 2}\right)$ peaks have been presented. The XPS measurements were performed on powdered ground pellets. In considering past reports on $\mathrm{BB}$ in the literature, the photoemission studies performed by various groups are not in agreement. Jeon et al. and Kulkarni et al. reported the presence of multiple $\mathrm{Bi} 4 \mathrm{f}$ lines which they attributed to multiple valence states of bismuth. ${ }^{25,26}$ Several other groups have reported narrow bismuth core level spectra. ${ }^{27-30}$ Some attributed it to the presence of just one bismuth state in the compound and some suggested that the level of disproportionation was too small to be detected by photoemission. As is evident in the Bi4f spectra presented in Fig. 2, a shoulder is clearly present on the high binding energy side of the Bi4f peaks. As determined by peak fitting, the most intense component of the $\mathrm{Bi}_{4 / 2}$ peak was at $157.7 \mathrm{eV}$; the higher binding shoulder was at $158.9 \mathrm{eV}$. These binding energies, and their separation, are close to those described in literature. ${ }^{25}$ The peaks used in the fit were symmetric, and the relative intensities of each pair of $\mathrm{Bi}_{42}$ and $\mathrm{Bi} 4 \mathrm{f}_{5 / 2}$ peaks were constrained to be $4: 3$. The widths of the peaks corresponding to the +5 state were slightly larger than those for the +3 state. In summary, the valence state of the target ion is directly proportional to the binding energy, and therefore, these shoulders can be attributed to the presence of $\mathrm{Bi}^{5+}$ in the sample, and therefore the data here clearly show evidence of the presence of multiple valence states for bismuth in the spectra for BB ceramics.

Figure 3 shows the dielectric permittivity and loss data for $\mathrm{BB}$ over the temperature range of approximately $-150^{\circ} \mathrm{C}$ to $200^{\circ} \mathrm{C}$. Past reports on $\mathrm{BB}$ suggest a phase transition from monoclinic $I 2 / m$ to rhombohedral $R \overline{3}$ at around $132^{\circ} \mathrm{C},{ }^{31,32}$ however, this could not be observed in Fig. 3 due to the effects of conduction losses. The dielectric permittivity values were high over the entire temperature range of measurement, again an artifact of high losses. A frequency-dependent relaxation was observed in the dielectric loss at sub-zero temperatures and the temperature of the relaxation peak maxima increased with increasing frequency. Lee et al. observed a 


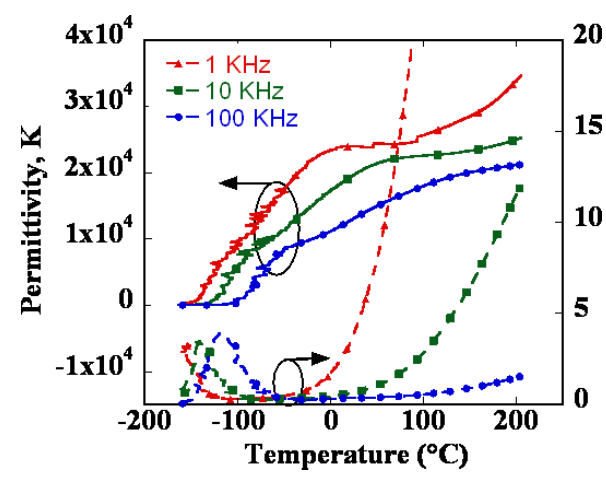

(a)

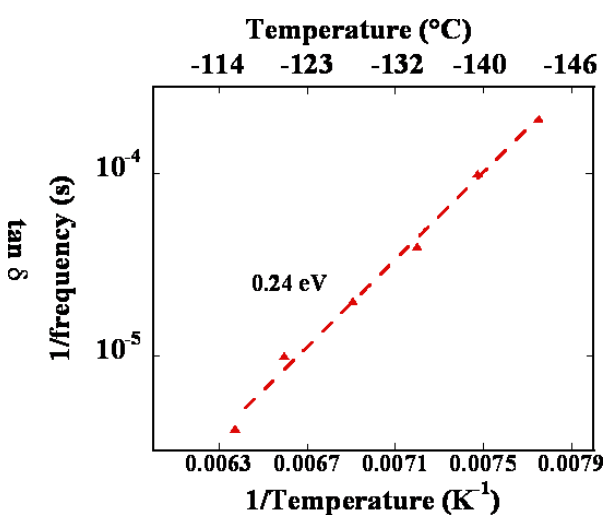

(b)

Fig. 3. (a) Permittivity and dielectric loss as a function of temperature at 1,10 and $100 \mathrm{kHz}$ for BB ceramics, and (b) Arrhenius plot of applied frequency and $1 / T_{\mathrm{m}}$, where $T_{\mathrm{m}}$ are the temperatures at dielectric tangent maximum.

similar thermally activated phenomenon in their study and attributed it to polaron hopping. ${ }^{33}$ Debye theory can be used to analyze the dielectric relaxation involving polaron hopping. An Arrhenius plot of the frequency and the temperature of relaxation peak yielded the activation energy for relaxation as shown in Fig. 3(b). ${ }^{33,34}$ The activation energy calculated in Fig. 3(b) was approximately $0.24 \mathrm{eV}$, which was consistent with literature reports. ${ }^{26}$ It was also close to the activation energy for conduction calculated through $a c$ impedance spectroscopy $(\sim 0.25 \mathrm{eV}$, Fig. 4(c)), which may suggest that similar mechanisms control both the dielectric relaxation and conduction mechanism.

The Kubelka-Munk function and Tauc plot were used to calculate the indirect and direct bandgaps, which were estimated to be $\sim 0.92 \mathrm{eV}$ and $\sim 2.2 \mathrm{eV}$, respectively (Figs. 4(a) and 4(b)). ${ }^{35-37}$ These values were consistent with literature values. ${ }^{38,39}$ It is worth mentioning that previous literature reports have largely failed to directly assess the indirect bandgap through techniques like optical reflectance, photoconductivity, photoacoustic spectroscopy, and other techniques.
The value of $0.92 \mathrm{eV}$ reported here is based on the assumption that the rise in the intensity of the signal close to $1 \mathrm{eV}$ in Fig. 4(b) was due to the indirect bandgap in BB. The presence of these bandgaps makes $\mathrm{BB}$ an indirect bandgap semiconductor. Band-structure calculations have predicted that BB should be metallic if bismuth were present in the 4+ state as it would have a half-filled $\mathrm{Bi} 6 s$ conduction band. ${ }^{40,41}$ The origin of semiconduction in BB is believed to be due to result of the disproportionation of bismuth into $3+$ and $5+$ valence states, followed by ordering and accompanied by alternate expansion and contraction of oxygen octahedra. In other words, the strong soft-phonon related electron-phonon interaction, which is considered to be responsible for superconductivity in BB-based ceramics, is completely softened leading to static displacement of the oxygen atoms in undoped BB. This, in turn, leads to a configuration called charge density wave (CDW) or breathing mode distortion, and is believed to be responsible for the observed indirect bandgap at the Fermi level, which then gives rise to semiconducting properties. ${ }^{31,33}$

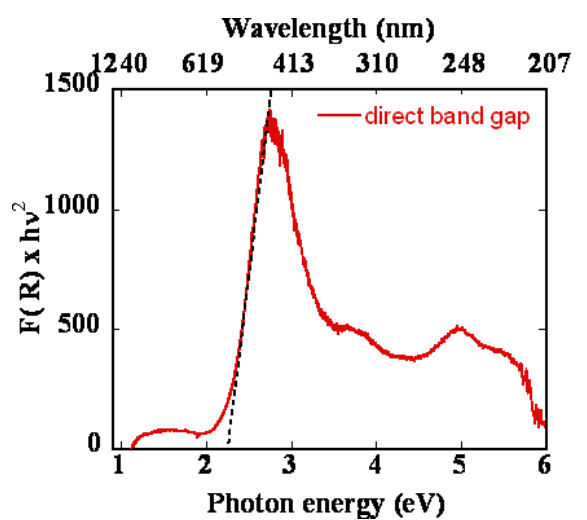

(a)

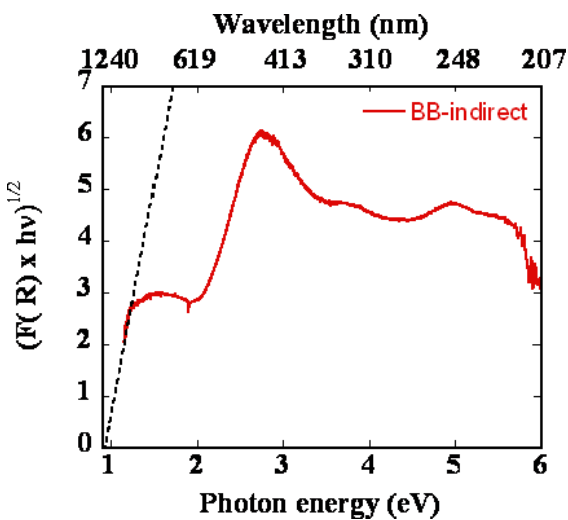

(b)

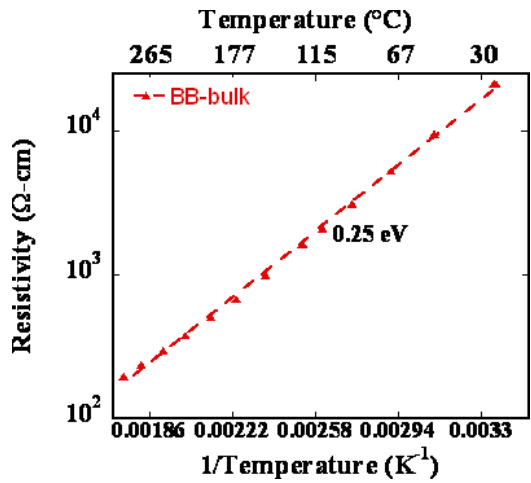

(c)

Fig. 4. Kubelka-Munk and Tauc plots for BB ceramics. The appropriate functions for direct and indirect bandgaps were chosen for (a) and (b) respectively. (a) and (b) also show representative fit lines used to estimate bandgaps. (c) shows Arrhenius plot with linear fit for bulk resistivity obtained from $a c$ impedance spectroscopy. 


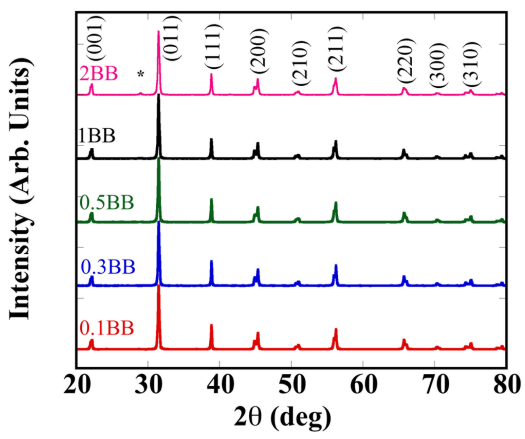

Fig. 5. XRD pattern of $(1-x) \mathrm{BT}-x \mathrm{BB}$ ceramics, where $x$ lies between $0.1 \mathrm{~mol} \%$ and $2 \mathrm{~mol} \%$. The " $*$ " indicates secondary phase, if present.

Figure 4(b) shows the activation energy for bulk conduction for $\mathrm{BB}$, calculated using $a c$ impedance spectroscopy. Impedance spectroscopy is a powerful tool and can effectively separate the bulk transport properties from other contributions such as interfaces, inhomogeneity, and other mechanisms. ${ }^{42}$ On plotting the bulk resistivity as a function of temperature using the Arrhenius equation, the conduction was shown to be a thermally activated process with an activation energy estimated to be $\sim 0.25 \mathrm{eV}$. This is consistent with the activation energy values reported using Seebeck and Hall measurements. ${ }^{38}$ This activation energy value is significantly lower than the measured bandgap values reported above. However, it has been suggested that this is closely related to the indirect bandgap and not related to impurities. ${ }^{41}$ It was also supported by the fact that the number of states contributing to electrical conduction calculated by using Hall measurements by Takagi et al. was comparable to the number of bismuth atoms in the crystal structure. ${ }^{43}$ However, to explain the low activation energy (as compared to bandgap measurements), it has been suggested that polaronic bands accommodating holes are formed above the top of occupied Bi $6 s$ band $(\sim 0.25 \mathrm{eV}$ below Fermi level) and a polaron band accommodating electrons are formed below the empty Bi $6 s$ band $\left(\sim 0.25 \mathrm{eV}\right.$ above Fermi level) ${ }^{41}$ Due to these polaron bands, the conductivity gap is reduced to $\sim 0.5 \mathrm{eV}$, which can explain the measured activation energy of $0.25 \mathrm{eV}$ obtained from impedance spectroscopy.

\subsection{BB-BT ceramics}

Following the results of the synthesis and characterization of pure $\mathrm{BB}$, this section aims to examine the change in transport properties on adding small amounts of BB to BT. Figure 5 shows the XRD patterns of pulverized BT-BB solid solution ceramics. All the samples had split $\{200\}$ peaks $\left(\sim 45^{\circ}\right)$, indicating tetragonal symmetry. Within the resolution of laboratory XRD, it was difficult to note any change in lattice parameters with the addition of such small amounts of BB. Notably, the sintering temperatures get reduced significantly (by $300^{\circ} \mathrm{C}-400^{\circ} \mathrm{C}$ ) on adding BB to BT. Still, all sintered pellets had measured density values greater than $95 \%$ of the theoretical value, as measured via Archemedes' method. It can also be noted in Fig. 5 that phase-pure perovskite could be achieved for BB-BT solid solutions with less than $2 \mathrm{~mol} \%$ BB. The secondary phase shown for $98 \mathrm{BT}-2 \mathrm{BB}$ is believed to be $\mathrm{BB}$ itself as the peak position matched well with the most intense peak of BB (Fig. 1).

Figure 6 shows the dielectric constant as a function of temperature. All the compositions exhibited a sharp phase transition as expected for a normal ferroelectric material. The Curie point was recorded to be between $122^{\circ} \mathrm{C}$ and $125^{\circ} \mathrm{C}$ for all compositions, and was in contrast with a previous report which claimed an increase in Curie point by $\sim 30^{\circ} \mathrm{C}$ on adding $\sim 0.5 \% \mathrm{BB}$ to $\mathrm{BT} .{ }^{17}$ The room temperature dielectric constant varied between $\sim 2000$ and $\sim 3000$. Notably, the dielectric loss values remained low $(<5 \%)$ up to $\sim 300^{\circ} \mathrm{C}$ at $1 \mathrm{kHz}$ for all compositions, which was better than BB (Fig. 3) or $\mathrm{BT}^{44}$ alone.

In order to further investigate long-range conduction in these ceramics, $a c$ impedance spectroscopy was performed and Fig. 7(a) shows the Arrhenius plots of the bulk resistivity values obtained from the same. The data for undoped BT and BB have also been shown for reference. In the plots, the BT$\mathrm{BB}$ ceramics appear to have multiple orders of magnitude higher resistivity than either $\mathrm{BT}$ or $\mathrm{BB}$ alone. The activation energy for conduction, estimated from the slopes of the plots, also increased significantly for the solid solution as compared to the end-member compounds. This suggests a dramatic change in the underlying defect chemistry in these solid solutions, which warrants further examination. The undoped BT used in this study is characterized by a defect-dominated p-type conduction mechanism, as reported in a previous paper. ${ }^{45}$ In BT, the p-type behavior is commonly attributed to frozen-in cation vacancies formed during high temperature processing and low valence acceptor impurities associated with starting reagent oxides. $^{46-48}$ These vacancies and acceptors can be compensated by oxygen vacancies, which can in turn give rise to p-type behavior at high oxygen partial pressures as per following defect reaction:

$$
\mathrm{V}_{\mathrm{O}}^{\ddot{*}}+\frac{1}{2} \mathrm{O}_{2} \rightarrow \mathrm{O}_{\mathrm{O}}^{\mathrm{X}}+2 \mathrm{~h}
$$

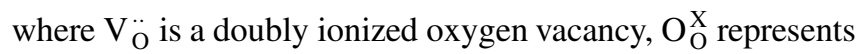
an oxygen ion located on the anion sublattice, and h represents a hole. In considering the significant change in conductivity with the addition of $\mathrm{BB}$, a number of factors could be responsible. First, the concentration of cation vacancies in BT-BB ceramics may be lower than BT alone owing to the significantly lower sintering temperatures (i.e., $1350^{\circ} \mathrm{C}$ for BT and $950-1050^{\circ} \mathrm{C}$ for BT-BB). Secondly, there may be an unintended donor dopant species present which is compensating for the holes and thus effectively increasing the resistivity values. One of the many possible candidates for unintentional donor doping could be the occupation of 


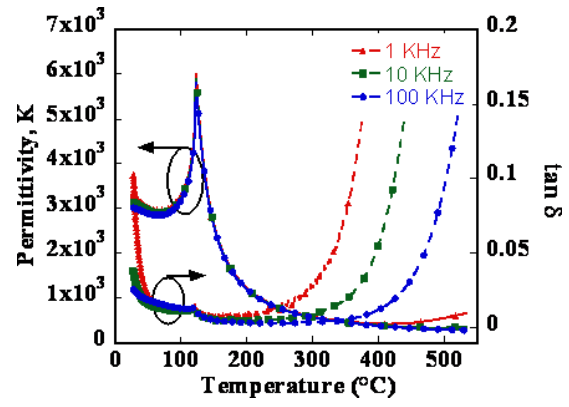

(a)

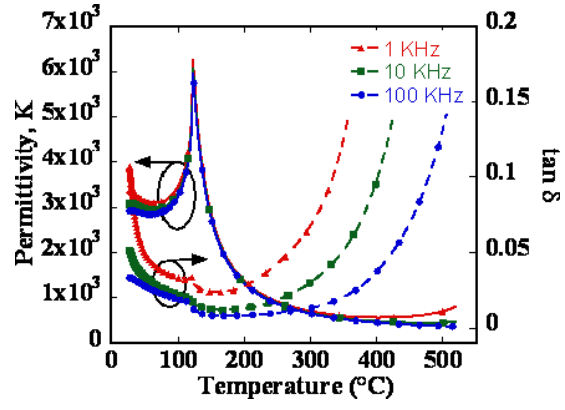

(b)

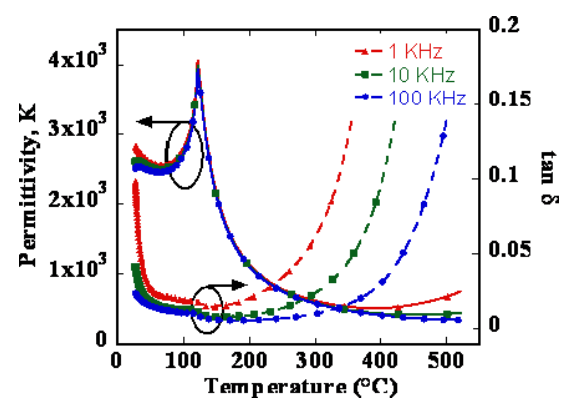

(c)

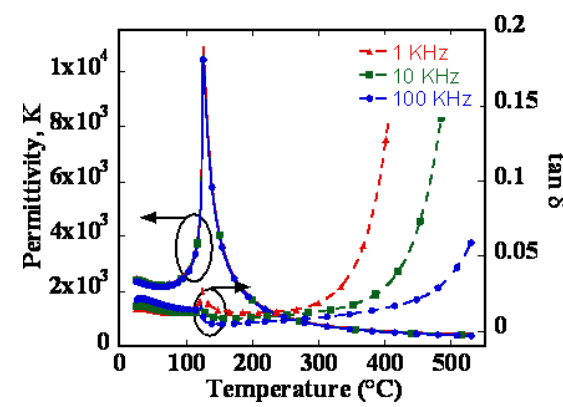

(d)

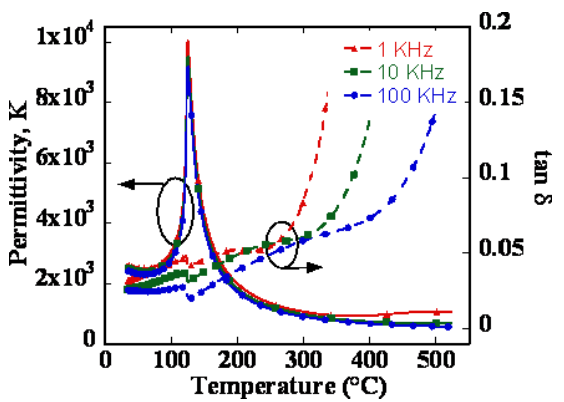

(e)

Fig. 6. Permittivity and dielectric loss as a function of temperature at 1,10 and $100 \mathrm{kHz}$ for (a) BT-0.1BB, (b) BT-0.3BB, (c) BT-0.5BB, (d) $\mathrm{BT}-1 \mathrm{BB}$, and (e) BT-2BB ceramics.

bismuth cations on the A-sublattice, where by replacing barium it will act as a donor. This possibility will be discussed later in this paper.

Figure 7(b) shows the Tauc plots using the KubelkaMunk function, as was shown for undoped BB ceramics earlier. The indirect bandgaps for all compositions were $\sim 3.1 \mathrm{eV}$, which is close to the value expected for titanatebased perovskites and is consistent with previous literature reports. ${ }^{44}$ The peculiar characteristic present in all these plots were the Urbach tail-like features, which became more apparent with increasing BB concentrations. Generally, it is considered to originate from disorder in a crystal such as localized defects, lattice vibrations, and other factors. ${ }^{36}$ These features lead to electronic states just above the valence band or immediately below the conduction band, and the transition between these bandtail states may give rise to Urbach tails. More details about these Urbach tails can be found elsewhere ${ }^{49,50}$ Another observation which can be made is that the extrapolations of these tails intercept at the $x$-axis at $\sim 1.8-2.2 \mathrm{eV}$, which is close to the value of direct bandgap

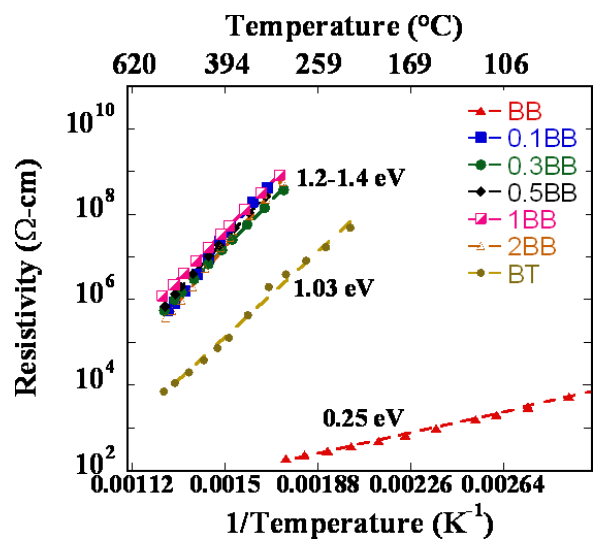

(a)

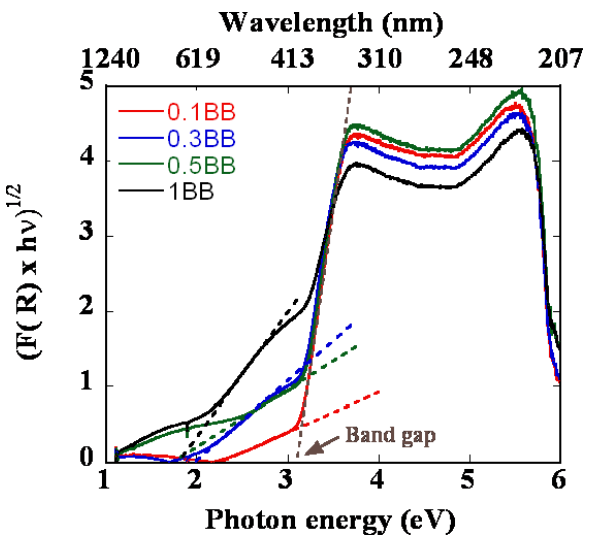

(b)

Fig. 7. (a) Arrhenius plots for bulk resistivity obtained from $a c$ impedance spectroscopy for BT-BB ceramics. Plots for undoped BB and BT have been shown for reference. (b) Kubelka-Munk and Tauc plots for BT-BB ceramics. The appropriate function for indirect bandgap was chosen. Representative fit lines have also been shown. 


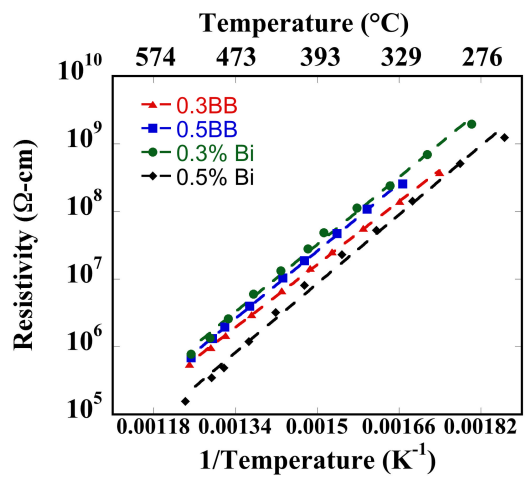

Fig. 8. Arrhenius plots for bulk resistivity obtained from $a c$ impedance spectroscopy for Bi-doped BT ceramics. Data for BT-BB ceramics have been shown for reference.

for BB. Therefore, these features may also be a direct result of a secondary BB phase which may be undetectable by laboratory scale XRD techniques.

In considering the mechanism for the increase in resistivity for BT-BB ceramics as compared to BT, unintentional donor doping due to changes in bismuth site occupancy was proposed to be a possibility. Direct confirmation of bismuth on the A-site is extremely challenging due to the relatively low concentration of bismuth. Therefore, an indirect approach has been adopted here to characterize the site occupancy of bismuth. In this study, BT ceramics were prepared with excess additions of $0.3 \mathrm{~mol} \%$ bismuth $(\mathrm{BT}+0.15 \%$ $\left.\mathrm{Bi}_{2} \mathrm{O}_{3}\right)$ and $0.5 \mathrm{~mol} \%$ bismuth $\left(\mathrm{BT}+0.25 \% \mathrm{Bi}_{2} \mathrm{O}_{3}\right)$ so that the amount of bismuth added was same as in the solid solutions BT- $0.3 \mathrm{BB}$ and $\mathrm{BT}-0.5 \mathrm{BB}$, respectively. These compounds were intentionally sintered under the same conditions as the BT-BB ceramics so as to make a direct comparison. It is believed that under normal circumstances, excess bismuth would substitute onto the A-sublattice to replace barium due to thermodynamic considerations and this has also been confirmed through multiple reports in the literature with electrical property measurements. ${ }^{50-52}$ The results from $a c$ impedance spectroscopy performed on them have been presented in Fig. 8. The Arrhenius plot for the bulk resistivity shows that the resistivity values of BT doped with bismuth was quite similar to BT-BB ceramics with similar bismuth content. The slopes were also almost identical, indicating similar activation energies for conduction. These results, therefore, suggest that it is the occupancy of bismuth on the A-site that leads to the observed improvement in resistivity.

\section{Conclusions}

Single-phase BB ceramics were fabricated and confirmed by high-resolution XRD, and the coexistence of bismuth in $3+$ and $5+$ valence states was confirmed by XPS. Using $a c$ impedance and optical spectroscopy, it was shown that polaron-hopping was the dominant conduction mechanism in BB. Ceramics based on the solid solution of BT-BB were fabricated to investigate the effect of adding small amounts of $\mathrm{BB}$ on the conduction mechanism of BT. It was observed that the BT-BB ceramics had significantly higher resistivity values as compared to undoped $\mathrm{BT}$ or $\mathrm{BB}$, suggesting a decrease in the concentration of mobile defect species. The resistivity values remained high even with a coexisting secondary BB phase. This is important for high temperature and high energy density capacitor applications. Data from $a c$ impedance spectroscopy on Bi-doped BT suggested that a shift in the site occupancy of Bi onto the A-sublattice is the dominant mechanism resulting in donor doping which results in the observed high resistivities.

\section{Acknowledgments}

This material is based upon work supported by the National Science Foundation under Grant No. DMR-1308032. Use of the Advanced Photon Source at Argonne National Laboratory was supported by the U. S. Department of Energy, Office of Science, Office of Basic Energy Sciences, under Contract No. DE-AC02-06CH11357. Thanks to Fallon Fumasi for her help with XPS measurements.

\section{References}

${ }^{1}$ A. W. Sleight, J. L. Gillson and P. E. Bierstedt, High-temperature superconductivity in the $\mathrm{BaPb}_{1-x} \mathrm{Bi}_{x} \mathrm{O}_{3}$ systems, Solid State Commun. 17, 27 (1975).

${ }^{2}$ W. R. Flavell, B. C. Morris, I. Tweddell, D. Purdie, G. Thornton, P. L. Wincott and R. L. Bilsborrow, Materials chemistry communications. $\mathrm{Pb}$ and $\mathrm{Bi}$ valencies in $\mathrm{BaPb}_{1-x} \mathrm{Bi}_{x} \mathrm{O}_{3}$, J. Mater. Chem. 2(11), 1209 (1992).

${ }^{3}$ Q. Zhou and B. J. Kennedy, High temperature structure of $\mathrm{BaBiO}_{3}$ - A synchrotron X-ray powder diffraction study, Solid State Commun. 132(6), 389 (2004).

${ }^{4}$ A. Glazer, The classification of tilted octahedra in perovskites, Acta Crystallogr. B: Struct. Crystallogr. Cryst. Chem. 28(11), 3384 (1972).

${ }^{5}$ C. J. Howard, B. J. Kennedy and P. M. Woodward, Ordered double perovskites-A group-theoretical analysis, Acta Crystallogr. B: Struct. Sci. 59(4), 463 (2003).

${ }^{6}$ A. Sleight, J. Gillson and P. Bierstedt, High-temperature superconductivity in the $\mathrm{BaPb}_{1-x} \mathrm{Bi}_{x} \mathrm{O}_{3}$ system, Solid State Commun. 17(1), 27 (1975).

${ }^{7}$ L. Mattheiss, E. Gyorgy and D. Johnson Jr, Superconductivity above $20 \mathrm{~K}$ in the Ba-K-Bi-O system, Phys. Rev. B 37(7), 3745 (1988).

${ }^{8}$ J. P. Attfield, Chemistry and high temperature superconductivity, J. Mater. Chem. 21(13), 4756 (2011).

${ }^{9}$ M. J. Akhtar and C. R. A. Catlow, X-Ray absorption studies of bibased superconductors, J. Mater. Chem. 4(7), 1081 (1994).

${ }^{10}$ M. Itoh, T. Sawada, R. Liang, H. Kawaji and T. Nakamura, Oxygen-deficient and ordered perovskite-type solid-solution system $\mathrm{Ba}_{1+x} \mathrm{Bi}_{1-x} \mathrm{O}_{y}(0 \leqq x \leqq 0.5,3.00 \geqq y \geqq 2.75)$, J. Solid State Chem. 87(1), 245 (1990).

${ }^{11}$ M. Hervieu, C. Michel, M. Caldes, A. Pham and B. Raveau, The cuprate $\mathrm{Bi}_{6} \mathrm{Ba}_{4} \mathrm{Cu}_{2} \mathrm{O}_{15}$ : A double collapsed 2201-type structure, J. Solid State Chem. 107(1), 117 (1993).

${ }^{12}$ J. Darriet, C. Dussarrat, F. Weill, B. Darriet and R. Bontchev, The system $\mathrm{BaRuO}_{3}-\mathrm{BaBiO}_{3}$. I. Crystal structures of $\mathrm{Ba}_{3} \mathrm{Ru}_{2} \mathrm{BiO}_{9}$ and 
$\mathrm{Ba}_{2} \mathrm{Ru}_{x} \mathrm{Bi}_{2-x} \mathrm{O}_{6}(0<x . L E Q .0 .67)$, Eur. J. Solid State Inorg. Chem. 30(3), 273 (1993).

${ }^{13}$ T. E. Sutto and B. A. Averill, Composition-and annealing-dependent properties of $\mathrm{Ba}(\mathrm{Bi}, \mathrm{Tl}) \mathrm{O}_{3}-\delta$, J. Solid State Chem. 105 (1), 263 (1993).

${ }^{14}$ J. Boyce, F. Bridges, T. Claeson, T. Geballe and J. Remeika, X-ray absorption of $\mathrm{BaBiO}_{3}$ and superconducting $\mathrm{BaBi}_{0.25} \mathrm{~Pb}_{0.75} \mathrm{O}_{3}$, Phys. Rev. B 41(10), 6306 (1990).

${ }^{15}$ Y. Luo, X. Liu, X. Li and G. Liu, PTCR effect in $\mathrm{BaBiO}_{3}$-doped $\mathrm{BaTiO}_{3}$ ceramics, Solid State Ionics 177(17), 1543 (2006).

${ }^{16} \mathrm{Q}$. Yuan, Y. Pu and B. Cao, Effects of different bismuth-based compounds additives on the positive temperature coefficient effect of $\mathrm{BaTiO}_{3}$ ceramics, Mater. Lett. 86, 115 (2012).

${ }^{17}$ Y. Chang-Lai, L. Xin-Yu, Z. Chang-Rong, X. Ji-Wen and Y. Yun, Characterization of the $\mathrm{BaBiO}_{3}$-doped $\mathrm{BaTiO}_{3}$ positive temperature coefficient of a resistivity ceramic using impedance spectroscopy with $\mathrm{T}_{\mathrm{c}}=155^{\circ} \mathrm{C}$, Chinese Phys. B 20(4) 048701 (2011).

${ }^{18}$ N. Kumar, A. Ionin, T. Ansell, S. Kwon, W. Hackenberger and D. Cann, Multilayer ceramic capacitors based on relaxor $\mathrm{BaTiO}_{3}-\mathrm{Bi}\left(\mathrm{Zn}_{1 / 2} \mathrm{Ti}_{1 / 2}\right) \mathrm{O}_{3}$ for temperature stable and high energy density capacitor applications, Appl. Phys. Lett. 106(25), 252901 (2015).

${ }^{19}$ C.-C. Huang and D. P. Cann, Phase transitions and dielectric properties in $\mathrm{Bi}\left(\mathrm{Zn}_{1 / 2} \mathrm{Ti}_{1 / 2}\right) \mathrm{O}_{3}-\mathrm{BaTiO}_{3}$ perovskite solid solutions, J. Appl. Phys. 104(2), 024117 (2008).

${ }^{20} \mathrm{~N}$. Raengthon and D. P. Cann, High temperature electronic properties of $\mathrm{BaTiO}_{3}-\mathrm{Bi}\left(\mathrm{Zn}_{1 / 2} \mathrm{Ti}_{1 / 2}\right) \mathrm{O}_{3}-\mathrm{BiInO}_{3}$ for capacitor applications, J. Electroceram. 28(2-3), 165 (2012).

${ }^{21}$ N. Triamnak, G. L. Brennecka, H. J. Brown-Shaklee, M. A. Rodriguez and D. P. Cann, Phase formation of $\mathrm{BaTiO}_{3}-\mathrm{Bi}\left(\mathrm{Zn}_{1 / 2} \mathrm{Ti}_{1 / 2}\right)$ $\mathrm{O}_{3}$ perovskite ceramics, J. Ceram. Soc. Jpn. 122(1424), 260 (2014).

${ }^{22}$ B. H. Toby and R. B. Von Dreele, GSAS-II: The genesis of a modern open-source all purpose crystallography software package, J. Appl. Crystallogr. 46(2), 544 (2013).

${ }^{23} \mathrm{D}$. Cox and A. Sleight, Crystal structure of $\mathrm{Ba}_{2} \mathrm{Bi}_{3+} \mathrm{Bi}_{5+} \mathrm{O}_{6}$, Solid State Commun. 19(10), 969 (1976).

${ }^{24}$ K. P. Reis, A. J. Jacobson and J. M. Nicol, A powder neutron diffraction investigation of structure and cation ordering in $\mathrm{Ba}_{2+x}$ $\mathrm{Bi}_{2-x} \mathrm{O}_{6-y}$, J. Solid State Chem. 107(2), 428 (1993).

${ }^{25}$ G. Kulkarni, V. Vijayakrishnan, G. R. Rao, R. Seshadri and C. Rao, State of bismuth in $\mathrm{BaBiO}_{3}$ and $\mathrm{BaBi}_{1-x} \mathrm{~Pb}_{x} \mathrm{O}_{3}$ : $\mathrm{Bi}$ 4f photoemission and $\mathrm{BiL}_{3}$ absorption spectroscopic studies, Appl. Phys. Lett. 57(17), 1823 (1990).

${ }^{26}$ Y. Jeon, G. Liang, J. Chen, M. Croft, M. Ruckman, D. Di Marzio and $\mathrm{M}$. Hegde, $\mathrm{BaBiO}_{3}$ and the effect of potassium substitution using photoemission, Phys. Rev. B 41(7), 4066 (1990).

${ }^{27} \mathrm{G}$. Wertheim, J. Remeika and D. Buchanan, Electronic structure of $\mathrm{BaPb}_{1-x} \mathrm{Bi}_{x} \mathrm{O}_{3}$, Phys. Rev. B 26(4), 2120 (1982).

${ }^{28} \mathrm{P}$. Ganguly and M. Hegde, Evidence for double valence fluctuation in metallic oxides of lead, Phys. Rev. B 37(10), 5107 (1988).

${ }^{29}$ M. Hegde, P. Barboux, C. Chang, J. Tarascon, T. Venkatesan, X. $\mathrm{Wu}$ and $\mathrm{A}$. Inam, Electronic structure of high- $\mathrm{T}_{c} \mathrm{Ba}_{0.6} \mathrm{~K}_{0.4} \mathrm{BiO}_{3}$ by X-ray photoelectron spectroscopy, Phys. Rev. B 39(7), 4752 (1989).

${ }^{30}$ Z.-X. Shen, P. Lindberg, B. Wells, D. Dessau, A. Borg, I. Lindau, W. Spicer, W. Ellis, G. Kwei and K. Ott, Photoemission study of monoclinic $\mathrm{BaBiO}_{3}$, Phys. Rev. B 40(10), 6912 (1989).

${ }^{31}$ D. Cox and A. Sleight, Mixed-valent $\mathrm{Ba}_{2} \mathrm{Bi}_{3+} \mathrm{Bi}_{5+} \mathrm{O}_{6}$ : Structure and properties vs temperature, Acta Crystallogr. B: Struct. Crystallogr. Cryst. Chem. 35(1), 1 (1979).
${ }^{32}$ F. Abbattista, M. Vallino, A. Delmastro, D. Mazza and S. Ronchetti, Research on the $\mathrm{BaBiO}_{3}-\delta$ System $(0 \leq \delta \leq 0.5), J$. Solid State Chem. 117(1), 55 (1995).

${ }^{33}$ S.-H. Lee, W.-H. Jung, J.-H. Sohn, J.-H. Lee and S.-H. Cho, Dielectric loss anomaly of $\mathrm{BaBiO}_{3}$, J. Appl. Phys. 86(11), 6351 (1999).

${ }^{34}$ L. Dominik and R. MacCrone, Dielectric relaxations in reduced rutile $\left(\mathrm{TiO}_{2-x}\right)$ at low temperatures, Phys. Rev. 163(3), 756 (1967).

${ }^{35}$ J. Klaas, G. Schulz-Ekloff and N. I. Jaeger, UV-visible diffuse reflectance spectroscopy of zeolite-hosted mononuclear titanium oxide species, J. Phys. Chem. B 101(8), 1305 (1997).

${ }^{36}$ J. Tauc, R. Grigorovici and A. Vancu, Optical properties and electronic structure of amorphous germanium, Physica Status Solidi (b) 15(2), 627 (1966).

${ }^{37}$ T. Umebayashi, T. Yamaki, H. Itoh and K. Asai, Band gap narrowing of titanium dioxide by sulfur doping, Appl. Phys. Lett. 81(3), 454 (2002).

${ }^{38}$ S. Tajima, S. Uchida, A. Masaki, H. Takagi, K. Kitazawa, S. Tanaka and $\mathrm{S}$. Sugai, Electronic states of $\mathrm{BaPb}_{1-x} \mathrm{Bi}_{x} \mathrm{O}_{3}$ in the semiconducting phase investigated by optical measurements, Phys. Rev. B 35(2), 696 (1987).

${ }^{39}$ K. H. Kim, C. Jung, T. Noh and S. Kim, Optical indirect transitions of semiconducting $\mathrm{BaPb}_{1-x} \mathrm{Bi}_{x} \mathrm{O}_{3}$, Phys. Rev. B 55(23), 15393 (1997).

${ }^{40} \mathrm{~L}$. Mattheiss and $\mathrm{D}$. Hamann, Electronic structure of $\mathrm{Ba} \mathrm{Pb}_{1-x}$ $\mathrm{Bi}_{x} \mathrm{O}_{3}$, Phys. Rev. B 28(8), 4227 (1983).

${ }^{41}$ H. Namatame, A. Fujimori, H. Takagi, S. Uchida, F. De Groot and J. Fuggle, Electronic structure and the metal-semiconductor transition in $\mathrm{BaPb}_{1-x} \mathrm{Bi}_{x} \mathrm{O}_{3}$ studied by photoemission and x-ray-absorption spectroscopy, Phys. Rev. B 48(23), 16917 (1993).

${ }^{42}$ B. Elissalde and J. Ravez, Ferroelectric ceramics: defects and dielectric relaxations, J. Mater. Chem. 11(8), 1957 (2001).

${ }^{43}$ H. Takagi, S. Uchida, S. Tajima, K. Kitazawa and S. Tajima, in Proc. Int. Conf. Physics of Semiconductors, ed. O. Engstrom, Stockholm (1986), p. 1815.

${ }^{44} \mathrm{~N}$. Kumar and D. P. Cann, Resistivity enhancement and transport mechanisms in (1-x) BaTiO ${ }_{3-x} \mathrm{Bi}\left(\mathrm{Zn}_{1 / 2} \mathrm{Ti}_{1 / 2}\right) \mathrm{O}_{3}$ and $(1-\mathrm{x}) \mathrm{SrTiO}_{3-x} \mathrm{Bi}$ $\left(\mathrm{Zn}_{1 / 2} \mathrm{Ti}_{1 / 2}\right) \mathrm{O}_{3}$, J. Am. Ceram. Soc. 98(8), 2548 (2015).

${ }^{45}$ N. Kumar, E. A. Patterson, T. Frömling and D. P. Cann, Conduction mechanisms in $\mathrm{BaTiO}_{3}-\mathrm{Bi}\left(\mathrm{Zn}_{1 / 2} \mathrm{Ti}_{1 / 2}\right) \mathrm{O}_{3}$ ceramics, J. Am. Ceram. Soc. 99(9), 3047-3054 (2016).

${ }^{46}$ N. H. Chan and D. Smyth, Defect chemistry of $\mathrm{BaTiO}_{3}$, J. Electrochem. Soc. 123(10), 1584 (1976).

${ }^{47}$ Y. Tsur and C. A. Randall, Point defect concentrations in barium titanate revisited, J. Am. Ceram. Soc. 84(9), 2147 (2001).

${ }^{48}$ R. Moos and K. H. Härdtl, Dependence of the intrinsic conductivity minimum of $\mathrm{SrTiO}_{3}$ ceramics on the sintering atmosphere, J. Am. Ceram. Soc. 78(9), 2569 (1995).

${ }^{49}$ Y. Chang, G. Badano, J. Zhao, Y. Zhou, R. Ashokan, C. Grein and V. Nathan, Near-bandgap infrared absorption properties of HgCdTe, J. Electron. Mater. 33(6), 709 (2004).

${ }^{50}$ H. Tran, W. Du, S. A. Ghetmiri, A. Mosleh, G. Sun, R. A. Soref, J. Margetis, J. Tolle, B. Li and H. A. Naseem, Systematic study of $\mathrm{Ge}_{1-x} \mathrm{Sn}_{x}$ absorption coefficient and refractive index for the device applications of Si-based optoelectronics, J. Appl. Phys. 119(10), 103106 (2016).

${ }^{51}$ D. Zhang, T. Wang, Y. Zhu, C. Wang and X. Wu, Some dielectric and photorefractive properties of $\mathrm{Bi}: \mathrm{BaTiO}_{3}$ and $\mathrm{Bi}, \mathrm{K}: \mathrm{BaTiO}_{3}$ crystals, Physica Status Solidi (a), 168(1), 297 (1998).

${ }^{52} \mathrm{P}$. Padmini and T. Kutty, Influence of Bi3+ ions in enhancing the magnitude of positive temperature coefficients of resistance in n-BaTiO 3 ceramics, J. Mater. Sci.: Mater. Electron. 5(4), 203 (1994). 